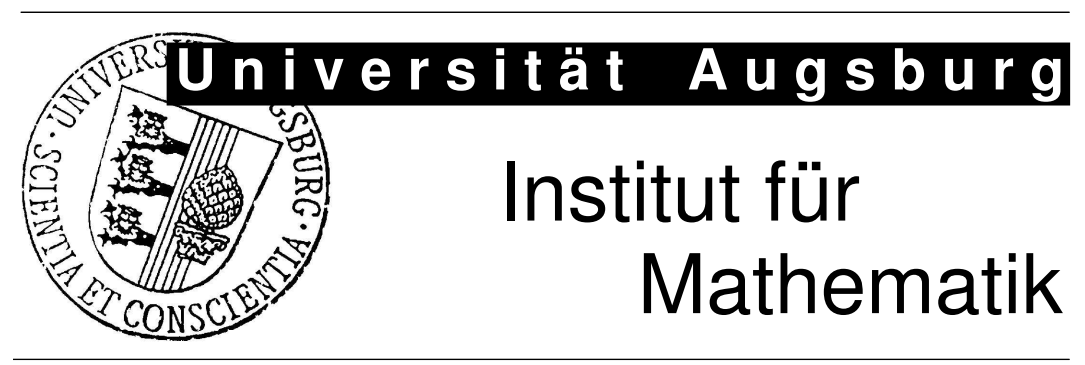

William G. Litvinov, Talal Rahman, Ronald H.W. Hoppe

Model of an Electro-Rheological Shock Absorber and Coupled Problem for Partial and Ordinary Differential Equationsl with Variable Unknown Domain 


\section{Impressum:}

Herausgeber:

Institut für Mathematik

Universität Augsburg

86135 Augsburg

http://www . math. uni-augsburg.de/forschung/preprint/

ViSdP:

Ronald H.W. Hoppe

Institut für Mathematik

Universität Augsburg

86135 Augsburg

Preprint: Sämtliche Rechte verbleiben den Autoren (C) 2007 


\title{
Model of an electro-rheological shock absorber and coupled problem for partial and ordinary differential equations with variable unknown domain.
}

\author{
W. G. LITVINOV ${ }^{1} \S$, T. RAHMAN ${ }^{2}$ and R. H. W. HOPPE ${ }^{3}$ \\ ${ }^{1}$ Institute of Mathematics, University of Augsburg, Universitätsstr. 14, D-86159 Augsburg, Germany \\ email: litvinov@math.uni-augsburg.de \\ 2 Department of Mathematics, University of Bergen, Allégaten 41, N-5007 Bergen, Norway \\ email: talal.rahman@math.uib.no \\ ${ }^{3}$ Department of Mathematics, University of Houston, Houston, TX 77204-3008, U.S.A., and \\ Institute of Mathematics, University of Augsburg, Universitätsstr. 14, D-86159 Augsburg, Germany \\ email: rohop@math.uh.edu
}

(Received 0000 0000)

\begin{abstract}
Amortization of a shock in an electrorheological shock absorber is carried out in the motion of a piston in an electrorheological fluid. The drag force acting on the piston is regulated by varying the voltage applied to electrodes. A model of an electrorheological shock absorber is constructed. A problem on shock absorber reduces to solution of a coupled problem for motion equation of the piston and nonlinear equations of fluid flow in an unknown domain that varies with the time. A method of semi-discretization for approximate solution of the coupled problem is considered. Results on the existence and on the uniqueness of the solution of the coupled problem are obtained. Convergence of approximate solutions to the exact solution is proved. Numerical simulation of the operation of the shock absorber is performed.
\end{abstract}

\section{Introduction}

Electrorheological fluids are smart materials which are concentrated suspensions of polarizable particles in a dielectric liquid. With an applied electric field the particles form chains aligned with the electric field. Such a unidirectional chainlike structure is anisotropic and it causes dramatic changes in the rheological properties of the fluids. The apparent viscosity (the resistance to flow) in the direction orthogonal to the direction of the electric field abruptly increases. It can increase by several orders of magnitude for electric fields of the orders of $1 \mathrm{kV} \mathrm{mm}^{-1}$, see [22]. The apparent viscosity of flow in the direction of the electric field also changes but not so drastically, see [26].

Due to their remarkable properties electrorheological fluids have various applications in electromechanical devices such as clutches, shock absorbers, valves and others [5]. Various problems on dynamics of electrorheological clutches and valves were formulated and studied in [6], [7], [32]-[35]. However, to the best of our knowledge, the problem of the electrorheological shock absorber has not been formulated and studied. Our paper is devoted to this problem.

A longitudinal section of an electro-rheological shock absorber is shown in Figure 1. As illustrated, the absorber consists of two fluid chambers filled with an electro-rheological fluid and a piston rod with two transfer ducts whose inner walls serve as electrodes. When a body strikes the piston, it moves, and the resistance to the motion of the piston is regulated by varying the voltage applied to the electrodes.

The flow of the fluid is presumed to be axially symmetric, and in line with Figure 1, we consider the domain of the flow which is displayed in cylindrical coordinates $r, z$ at the instants of time $t=0$

$\S$ Corresponding author 


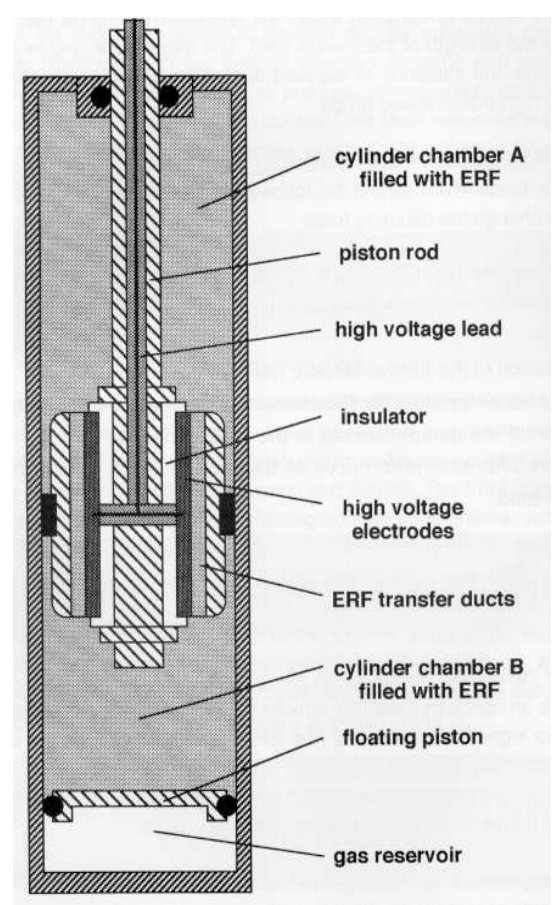

FIGURE 1. Schematic representation of an electro-rheological fluid (ERF) shock absorber.

and $t>0$ in Figure 2. The lightly shaded domain is the domain of the fluid, and the heavily shaded domain corresponds to the piston rod. The shape of the domain of flow is defined by the displacement of the piston $a(t)$, and we denote the domain of flow corresponding to the displacement $a(t)$ by $\Omega_{a(t)}$. In this case $a(0)=0$. Electrodes are placed on the parts $C D$ and $C^{\prime} D^{\prime}$ of the boundary of the domain of the fluid. When the piston rod is displaced by an amount $a(t)=l_{1}(t)-l_{1}(0)$, the floating piston is displaced from the origin by the amount $b(t)=a(t) R_{1}^{2} R^{-2}$ (see Figure 2) since the fluid is assumed to be incompressible.

Suppose that a solid body of a mass $m$ falls on the upper part of the piston with a velocity directed opposite to the direction of the $z$ axis, and the body moves along with the piston. According to the Newton principle, the motion of the piston satisfies

$$
m_{1} \frac{d v}{d t}(t)=G(t, v(t), V(t))
$$

Here $m_{1}=m+m_{0}, m_{0}$ is the mass of the piston, $v(t)$ the velocity of the piston, and $G(t, v(t), V(t))$ the drag force acting on the piston. The drag force depends on the applied voltage $V(t)$ and on the velocity $v(t)$. In this case $v$ is a scalar function satisfying the initial condition

$$
v(0)=v_{0}<0
$$

In the case that $m$ is considerably greater than $m_{0}$, one can consider that $v_{0}$ is the velocity of the falling body at the instant the body comes in contact with the piston. The drag force is computed by the solution of a problem for the electro-rheological fluid flow.

Below in Section 2, we formulate problems for the electric field and for the fluid flow. In this case the flow domain $\Omega_{a(t)}$ varies with time. The problem for the shock absorber reduces to solution of a coupled problem for the motion equation of the piston (1.1) and the equations of fluid flow in variable unknown domain $\Omega_{a(t)}$. The equations for the electric field and the velocity and pressure of the fluid are not coupled.

In Section 3, we present an operator formulation of the problem for the fluid flow. For the sake of 


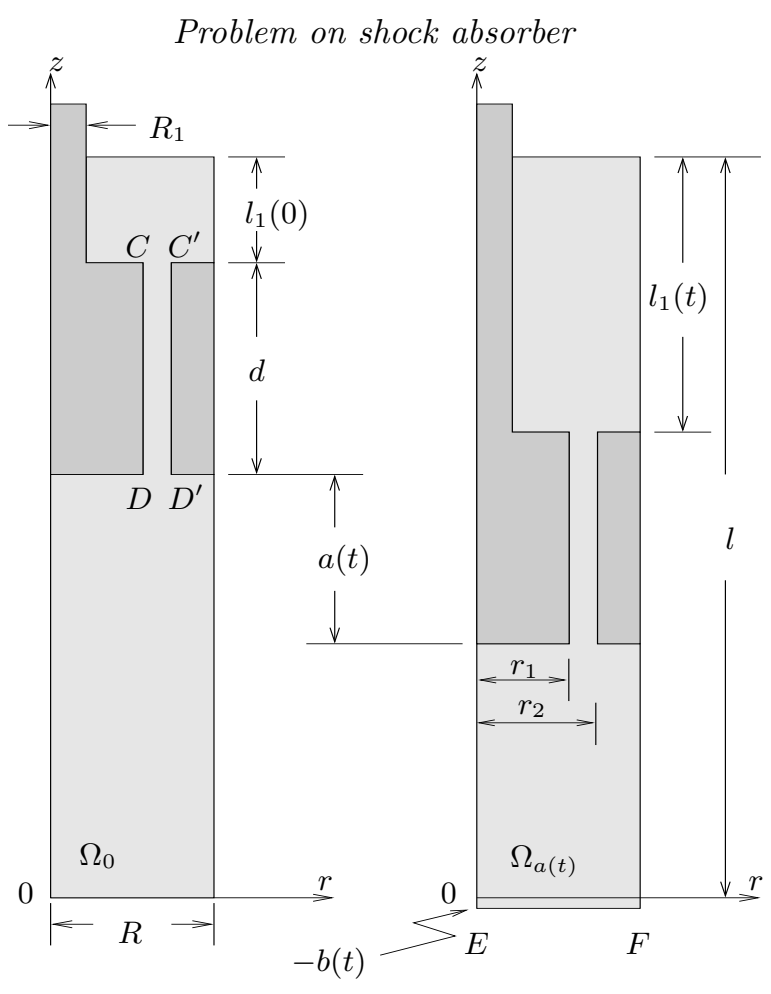

Figure 2. Domain of flow of the electro-rheological fluid at instants of time $t=0$ (left) and $t>0$ (right).

simplicity, it is assumed here that the inertial forces are equal to zero. We explain why this assumption is physically appropriate and acceptable in practice if the initial velocity of the piston is not very large. Moreover, it is pointed out that under neglect of the inertial forces, one obtains an upper bound of the time of absorption. This upper bound is very close to the time of absorption with regard for the inertial forces.

A theorem on the existence and on the uniqueness of the weak solution of the problem on fluid flow is presented for the case that the function $t \rightarrow a(t)$ is given. This theorem follows from the previous results of the authors and from known results of the theory of monotone operators.

In Section 4, we consider a method of semi-discretization for numerical solution of the coupled problem on shock absorber. The discretization is made with respect to the time variable. The closed interval of time $[0, T]$ is subdivided on $N$ parts, the time-step $k=T / N$ and $t_{n}=n k, n=1,2, \ldots, N$. The functions of velocity of the piston, velocity and pressure of the fluid, and electric field are assumed to be affine in each subinterval $\left[t_{n}, t_{n+1}\right]$. Having solved the problem on fluid flow at $t=t_{n}$ in $\Omega_{a\left(t_{n}\right)}$, the drag force is calculated and $\frac{d v}{d t}\left(t_{n}\right)$ is computed by (1.1), then $a\left(t_{n+1}\right)$ and $\Omega_{a\left(t_{n+1}\right)}$ are defined, $n=0,1,2, \ldots, N$.

Results on the existence and on the uniqueness of the solution of our coupled problem with variable domain and on convergence of approximate solutions obtained by the method of semi-discretization to the exact solution of the coupled problem are established in Section 5. A scheme of the proof of these results is the following: For each displacement of the piston $q=a(t)$ a homeomorphism $\mathcal{P}_{q}$ of the closed domain $\bar{\Omega}_{q}$ onto a fixed closed domain $\bar{\Omega}$ is constructed. The mappings $\mathcal{P}_{q}$ and the inverse of it $\mathcal{P}_{q}^{-1}$ are continuously differentiable almost everywhere in $\bar{\Omega}_{q}$ and in $\bar{\Omega}$, respectively.

The problems with variable domains are transformed to the problems with the fixed domain $\bar{\Omega}$. It is proved that the solutions obtained by the method of semi-discretization are bounded in corresponding norms as the parameter $k$ tends to zero, and these solutions depend continuously on $k$. Passing on to the limit, we obtain the results on the existence and on convergence.

Simulation results and conclusions are contained in Section 6 and 7, respectively. 
We mention that the methods of approximation and of proof of convergence of approximate solutions applied in Sections 4 and 5 are some modifications of the method developed in [21]. In the paper [21], the idea of delay is used for approximation of problems on plastic flow with moving unknown boundary.

Problems for the flow of viscous fluids with moving unknown boundary were investigated in many works, see e.g. [12], [23], [24], [25], [29], [30].

\section{Governing equations for the electro-rheological fluid.}

\subsection{Constitutive equation}

We consider the following constitutive equation of the electro-rheological fluid (see [13]):

$$
\sigma_{i j}(p, u, E)=-p \delta_{i j}+2 \varphi(I(u),|E|, \mu(u, E)) \varepsilon_{i j}(u), \quad i, j=1, \cdots, n, \quad n=2 \text { or } 3 .
$$

Here, $\sigma_{i j}(p, u, E)$ are the components of the stress tensor which depend on the pressure $p$, the velocity vector $u=\left(u_{1}, \cdots, u_{n}\right)$, and the electric field $E=\left(E_{1}, \cdots, E_{n}\right), \delta_{i j}$ are the components of the unit tensor (the Kronecker delta), and $\varepsilon_{i j}(u)$ are the components of the rate of strain tensor

$$
\varepsilon_{i j}(u)=\frac{1}{2}\left(\frac{\partial u_{i}}{\partial x_{j}}+\frac{\partial u_{j}}{\partial x_{i}}\right)
$$

where $x_{i}$ are Cartesian coordinates of a point $x=\left(x_{1}, \cdots, x_{n}\right)$. Moreover, $I(u)$ is the second invariant of the rate of strain tensor.

$$
I(u)=\sum_{i, j=1}^{n}\left(\varepsilon_{i j}(u)\right)^{2},
$$

and $\varphi$ is the viscosity function depending on $I(u),|E|$ and $\mu(u, E)$.

The function $\mu$ is introduced in the constitutive equation (2.1) in order to take into account the anisotropy of the electro-rheological fluid under which, the viscosity of the fluid depends on the angle between the vector of electrical field and the vector of velocity with respect to the charged electrode. The viscosity is maximal when the vector of velocity is orthogonal to the vector of electric field, and minimal when these vectors are parallel.

The electrode can move relative to the body of an electro-rheological device, and hence, we consider that the electrode can move relative to the reference frame under consideration. Let $x^{\prime}$ be a second reference frame which is immutably connected with the electrode, so that the electrode is immovable in the reference frame $x^{\prime}$. Let $\hat{u}(x, t)=\left(\hat{u}_{1}(x, t), \hat{u}_{2}(x, t), \hat{u}_{3}(x, t)\right)$ be a vector of transfer velocity, $\hat{u}(x, t)$ is a velocity of a point of the reference frame $x^{\prime}$ that coincides with the point $x$ of the first reference frame at an instant $t$. It is assumed that $\hat{u}$ is a known function.

We define the function $\mu$ as the square of the cosine of the angle between the vector of electric field and the vector of velocity relative to the electrode, i.e.

$$
\mu(u, E)=\left(\frac{u-\hat{u}}{|u-\hat{u}|}, \frac{E}{|E|}\right)_{\mathbb{R}^{3}}^{2}=\frac{\left(\left(u_{i}-\hat{u}_{i}\right) E_{i}\right)^{2}}{\left(u_{i}-\hat{u}_{i}\right)\left(u_{i}-\hat{u}_{i}\right) E_{j} E_{j}} .
$$

Here and below the Einstein convention on summation over repeated index is applied, and we denote by $(., .)_{\mathbb{R}^{3}}$ the scalar product in $\mathbb{R}^{3}$.

If the electrode does not move relative to the reference frame, then $\hat{u}=0$ and the function $\mu$ takes the form

$$
\mu(u, E)=\left(\frac{u}{|u|}, \frac{E}{|E|}\right)_{\mathbb{R}^{3}}^{2} .
$$

In the general case, the function $\hat{u}$ is defined as follows:

$$
\hat{u}(x, t)=\check{u}(t)+w(x, t),
$$


where $\check{u}(t)=\left(\check{u}_{1}(t), \check{u}_{2}(t), \check{u}_{3}(t)\right)$ is the vector of translation velocity, and $w(x, t)=\left(w_{1}(x, t)\right.$, $\left.w_{2}(x, t), w_{3}(x, t)\right)$ the vector of rotational velocity,

$$
\begin{aligned}
& w_{1}(x, t)=\omega_{2}(t) x_{3}-\omega_{3}(t) x_{2}, \\
& w_{2}(x, t)=\omega_{3}(t) x_{1}-\omega_{1}(t) x_{3}, \\
& w_{3}(x, t)=\omega_{1}(t) x_{2}-\omega_{2}(t) x_{1} .
\end{aligned}
$$

Here $\omega(t)=\left(\omega_{1}(t), \omega_{2}(t), \omega_{3}(t)\right)$ is the vector of angular velocity at an instant $t$.

The function $\mu(u, E)$ is an invariant which is independent of the choice of the reference frame and of the motion of the frame with respect to the electrode.

The viscosity function $\varphi$ is identified by approximation of flow curves (see [13]), and it was shown in [13] that it can be represented as follows:

$$
\varphi(I(u),|E|, \mu(u, E))=\zeta(|E|, \mu(u, E))(\lambda+I(u))^{-\frac{1}{2}}+\psi(I(u),|E|, \mu(u, E)),
$$

where $\lambda$ is a small parameter, $\lambda \geq 0$.

\subsection{The problem for the electric field.}

We consider our problem in the cylindrical coordinate system $(r, \alpha, z)$ and assume that the flow of the fluid is axially symmetric, i.e., the functions $u, p$, and $E$ depend only on $r, z, t$ in the mobile orthonormal basis $e_{r}, e_{\alpha}$ and $e_{z}$. The vectors $u$ and $E$ are of the forms $u(r, z, t)=\left(u_{r}(r, z, t), 0, u_{z}(r, z, t)\right)$ and $E(r, z, t)=\left(E_{r}(r, z, t), 0, E_{z}(r, z, t)\right)$. We use the notations $u_{r}=u_{1}, u_{z}=u_{2}, E_{r}=E_{1}$, and $E_{z}=E_{2}$, so that $u=\left(u_{1}, u_{2}\right)$, and $E=\left(E_{1}, E_{2}\right)$. We denote the value of the function $E$ at an instant $t$ by $E^{t}$, i.e., $E(r, z, t)=E^{t}(r, z)$, where $(r, z) \in \Omega_{a(t)}$.

Since electro-rheological fluids are essentially dielectrics, the magnetic fields in these fluids can be neglected. At each instant of time $t$ there exists a function of electric potential $\theta_{t}$ (see [13]) such that

$$
E^{t}=-\operatorname{grad} \theta_{t}
$$

and $\theta_{t}$ is the solution of the following problem:

$$
\begin{aligned}
\operatorname{div}\left(\chi \operatorname{grad} \theta_{t}\right) & =0 \text { in } \Omega_{a(t)}, \\
\theta_{t} & =V(t) \text { on } S_{1 a(t)}, \\
\theta_{t} & =0 \text { on } S_{0 a(t)}, \\
\frac{\partial \theta_{t}}{\partial r} & =0 \text { on } S_{2 a(t)}, \\
\nu_{1} \chi \frac{\partial \theta_{t}}{\partial r}+\nu_{2} \chi \frac{\partial \theta_{t}}{\partial z} & =0 \text { on } S_{a(t)} \backslash\left(\bigcup_{i=0}^{2} \bar{S}_{i a(t)}\right) .
\end{aligned}
$$

Here $\chi$ is the dielectric permittivity, $S_{1 a(t)}$ and $S_{0 a(t)}$ are the surfaces of the control and null electrodes respectively (lines $C D$ and $C^{\prime} D^{\prime}$ in the Figure 2), $S_{a(t)}$ is the boundary of $\Omega_{a(t)}, \nu_{1}$ and $\nu_{2}$ are the radial and axial components of the unit outward normal $\nu$ to the boundary $S_{a(t)}$.

In the case at hand, we have

$$
\begin{aligned}
S_{2 a(t)}=\left\{(r, z) \mid(r, z) \in \bar{\Omega}_{a(t)}, r=0\right\} & \\
& \operatorname{grad} \theta_{t}=\left(\frac{\partial \theta_{t}}{\partial r}, \frac{\partial \theta_{t}}{\partial z}\right),
\end{aligned}
$$


and equation (2.10) takes the form

$$
\frac{\partial}{\partial r}\left(\chi \frac{\partial \theta_{t}}{\partial r}\right)+\frac{\chi}{r} \frac{\partial \theta_{t}}{\partial r}+\frac{\partial}{\partial z}\left(\chi \frac{\partial \theta_{t}}{\partial z}\right)=0 \text { in } \Omega_{a(t)} .
$$

Let $q$ be a displacement of the piston. The displacement of the piston is limited, $q \in[0, \mathcal{A}]$, where $\mathcal{A}$ is an admissible displacement,

$$
\mathcal{A}=\frac{R^{2}\left(l-l_{1}(0)-d\right)}{R^{2}-R_{1}^{2}} .
$$

At $q=\mathcal{A}$ there is no fluid beneath the piston, i.e. in the lower chamber $B$.

For an arbitrary $q \in[0, \mathcal{A}]$, we define the following set:

$$
Z_{q}=\left\{w \in C^{\infty}\left(\bar{\Omega}_{q}\right), \frac{\partial w}{\partial r}=0 \text { on } S_{2 q}\right\}
$$

Here $\Omega_{q}$ is the domain of flow corresponding to the displacement $q$ of the piston.

Let $Z_{0 q}$ be the closure of $Z_{q}$ with respect to the norm

$$
\|w\|_{Z_{0 q}}=\left(\int_{\Omega_{q}}\left[w^{2}+\left(\frac{\partial w}{\partial r}\right)^{2}+\left(\frac{\partial w}{\partial z}\right)^{2}\right] r d r d z\right)^{\frac{1}{2}} .
$$

Again, we consider the following space:

$$
Z_{q}=\left\{w \mid w \in Z_{0 q}, w=0 \text { on } S_{0 q} \bigcup S_{1 q}\right\}
$$

The expression

$$
\|w\|_{Z_{q}}=\left(\int_{\Omega_{q}}\left[\left(\frac{\partial w}{\partial r}\right)^{2}+\left(\frac{\partial w}{\partial z}\right)^{2}\right] r d r d z\right)^{\frac{1}{2}}
$$

defines a norm in $Z_{q}$ being equivalent to the norm of $Z_{0 q}$ determined by (2.19).

Let $\theta_{0 t}$ be a function such that

$$
\theta_{0 t} \in Z_{0 a(t)}, \theta_{0 t}=V(t) \text { on } S_{1 a(t)}, \theta_{0 t}=0 \text { on } S_{0 a(t)} .
$$

We assume also that the function $\chi$ is given in a large domain $\Theta$ that contains all domains $\Omega_{q}, \chi$ is integrable in $\Theta$ with respect to the measure $r d r d z$ and, in addition,

$$
b_{1} \geq \chi \geq b_{0}>0 \text { a.e. in } \Theta
$$

where $b_{0}$ and $b_{1}$ are positive constants.

Define a bilinear form $e_{q}: Z_{0 q} \times Z_{q} \rightarrow \mathbb{R}$ as follows:

$$
e_{q}(f, h)=\int_{\Omega_{q}} \chi\left(\frac{\partial f}{\partial r} \frac{\partial h}{\partial r}+\frac{\partial f}{\partial z} \frac{\partial h}{\partial z}\right) r d r d z, \quad f \in Z_{0 q}, h \in Z_{q}
$$

Consider the problem: Find $\theta_{1 t}$ satisfying

$$
\theta_{1 t} \in Z_{a(t)}, e_{a(t)}\left(\theta_{1 t}, h\right)=-e_{a(t)}\left(\theta_{0 t}, h\right), \quad h \in Z_{a(t)} .
$$

The function $\theta_{t}=\theta_{0 t}+\theta_{1 t}$ is the weak solution of the problem (2.17),(2.11)-(2.14).

The Riesz theorem implies

Theorem 2.1 Suppose that the function $a(t)$ is given and the conditions (2.22), (2.23) are satisfied. Then there exists a unique weak solution $\theta_{t}$ of the problem (2.17),(2.11)-(2.14). The function $\theta_{t}$ is represented in the form $\theta_{t}=\theta_{0 t}+\theta_{1 t}$, where $\theta_{0 t}$ is a function satisfying (2.22) and $\theta_{1 t}$ is the solution of the problem (2.25). 


\subsection{The problem for the fluid flow}

In the case at hand, the components of the rate of strain tensor have the following form in cylindrical coordinates:

$$
\begin{gathered}
\epsilon_{11}(u)=\frac{\partial u_{1}}{\partial r}, \epsilon_{22}(u)=\frac{u_{1}}{r}, \epsilon_{33}(u)=\frac{\partial u_{2}}{\partial z}, \\
\epsilon_{13}(u)=\epsilon_{31}(u)=\frac{1}{2}\left(\frac{\partial u_{1}}{\partial z}+\frac{\partial u_{2}}{\partial r}\right), \\
\epsilon_{12}(u)=\epsilon_{21}(u)=\epsilon_{32}(u)=\epsilon_{23}(u)=0 .
\end{gathered}
$$

The second invariant of the rate of strain tensor is defined by

$$
I(u)=\left(\frac{\partial u_{1}}{\partial r}\right)^{2}+\left(\frac{u_{1}}{r}\right)^{2}+\left(\frac{\partial u_{2}}{\partial z}\right)^{2}+\frac{1}{2}\left(\frac{\partial u_{1}}{\partial z}+\frac{\partial u_{2}}{\partial r}\right)^{2} .
$$

Let $Q=\left\{(r, z, t) \mid t \in(0, T),(r, z) \in \Omega_{a(t)}\right\}$. The equations of motion have the following forms:

$$
\begin{gathered}
\rho\left(\frac{\partial u_{1}}{\partial t}+u_{1} \frac{\partial u_{1}}{\partial r}+u_{2} \frac{\partial u_{1}}{\partial z}\right)+\frac{\partial p}{\partial r} \\
-2 \frac{\partial}{\partial r}\left(\varphi \epsilon_{11}(u)\right)-2 \frac{\partial}{\partial z}\left(\varphi \epsilon_{13}(u)\right)-\frac{2}{r} \varphi\left(\epsilon_{11}(u)-\epsilon_{22}(u)\right)=K_{1} \text { in } Q, \\
\rho\left(\frac{\partial u_{2}}{\partial t}+u_{1} \frac{\partial u_{2}}{\partial r}+u_{2} \frac{\partial u_{2}}{\partial z}\right)+\frac{\partial p}{\partial r} \\
-2 \frac{\partial}{\partial r}\left(\varphi \epsilon_{13}(u)\right)-2 \frac{\partial}{\partial z}\left(\varphi \epsilon_{33}(u)\right)-\frac{2}{r} \varphi \epsilon_{13}(u)=K_{2} \text { in } Q .
\end{gathered}
$$

Here $K_{1}$ and $K_{2}$ are the radial and axial components of the vector of volume force $K=\left(K_{1}, K_{2}\right), \rho$ is the density, and the function $\varphi$ is defined by (2.8).

The equation of incompressibility has the form

$$
\operatorname{div} u=\frac{\partial u_{1}}{\partial r}+\frac{\partial u_{2}}{\partial z}+\frac{u_{1}}{r}=0 \text { in } Q .
$$

Denote the boundary of the piston at an instant $t$ by $S_{3 a(t)}$, and by $S_{4 a(t)}$ the lower part of the boundary $S_{a(t)}$ of $\Omega_{a(t)}$ (line EF in Figure 2, right).

Let

$$
\begin{aligned}
\Gamma & =\left\{(r, z, t) \mid t \in[0, T],(r, z) \in S_{a(t)}\right\}, \\
\Gamma_{2} & =\left\{(r, z, t) \mid t \in[0, T],(r, z) \in S_{2 a(t)}\right\}, \\
\Gamma_{3} & =\left\{(r, z, t) \mid t \in[0, T],(r, z) \in S_{3 a(t)}\right\}, \\
\Gamma_{4} & =\left\{(r, z, t) \mid t \in[0, T],(r, z) \in S_{4 a(t)}\right\} .
\end{aligned}
$$

We consider the following boundary conditions:

$$
\begin{gathered}
\left.u_{1}\right|_{\Gamma}=0,\left.u_{2}\right|_{\Gamma_{3}}=v,\left.u_{2}\right|_{\Gamma_{4}}=v R_{1}^{2} R^{-2}, \\
\left.\left.u_{2}\right|_{\Gamma \backslash\left(\bigcup_{i=2}^{4} \bar{\Gamma}_{i}\right)=0,} \frac{\partial u_{2}}{\partial r}\right|_{\Gamma_{2}}=0 .
\end{gathered}
$$

Here the condition on $\Gamma_{4}$ follows from the equation of incompressibility (2.30) under the assumption that the lower boundary of $\Omega_{a(t)}$ (line EF in Figure 2, right) is rigid. In this case the pressure in the gas reservoir enables one to determine uniquely the function of pressure of the fluid (see Subsection 3.2).

The initial condition has the form

$$
u(r, z, 0)=u_{0}(r, z) \text { in } \Omega_{a(0)} .
$$

The drag force $G(t, v(t), V(t))$ in (1.1) is defined as

$$
G(t, v(t), V(t))=-\int_{S_{3 a(t)}}\left[2 \varphi \epsilon_{31}(u) \nu_{1}+\left(-p+2 \varphi \epsilon_{33}(u)\right) \nu_{2}\right] d s .
$$


The problem on electro-rheological shock absorber consists in the finding of the piston velocity $v$, of the electric potential $\theta$, and of the velocity and pressure of the fluid $u$ and $p$, moreover, the domain $Q$ is unknown. The domain $\Omega_{a(t)}$ is defined by the displacement of the piston $a(t)$ (see Figure 2),

$$
a(t)=-\int_{0}^{t} v(\tau) d \tau,
$$

and $v(t)$ is the solution of the problem (1.1) and (1.2). In this case

$$
l_{1}(t)=l_{1}(0)-\int_{0}^{t} v(\tau) d \tau
$$

\section{Operator formulation of the problem on fluid flow.}

\subsection{Assumptions and spaces of functions}

Denote the values of the functions $u, p$, and $K$ at a point of time $t$ by $u^{t}, p^{t}$ and $K^{a(t)}$, i.e., $u(r, z, t)=$ $u^{t}(r, z), p(r, z, t)=p^{t}(r, z)$ and $K(r, z, t)=K^{a(t)}(r, z)$ where $(r, z) \in \Omega_{a(t)}$.

For the sake of simplicity, we ignore the inertial forces in the equations of fluid flow and consider the problem in the quasi-stationary formulation, i.e. we consider the following motion equations:

$$
\begin{gathered}
\frac{\partial p^{t}}{\partial r}-2 \frac{\partial}{\partial r}\left(\varphi \epsilon_{11}\left(u^{t}\right)\right)-2 \frac{\partial}{\partial z}\left(\varphi \epsilon_{13}\left(u^{t}\right)\right)-\frac{2}{r} \varphi\left(\epsilon_{11}\left(u^{t}\right)-\epsilon_{22}\left(u^{t}\right)\right)=K_{1}^{a(t)} \text { in } \Omega_{a(t)}, \\
\frac{\partial p^{t}}{\partial z}-2 \frac{\partial}{\partial r}\left(\varphi \epsilon_{31}\left(u^{t}\right)\right)-2 \frac{\partial}{\partial z}\left(\varphi \epsilon_{33}\left(u^{t}\right)\right)-\frac{2}{r} \varphi \epsilon_{13}\left(u^{t}\right)=K_{2}^{a(t)} \text { in } \Omega_{a(t)} .
\end{gathered}
$$

Here $\varphi$ is defined by the right-hand side of (2.8) with $u=u^{t}, E=E^{t}$, and it is presumed that the functions $a(t)$ and $E^{t}$ are given. Ignoring the inertial forces in (3.1) and (3.2) is physically appropriate and acceptable in practice if the initial velocity of the piston $v_{0}$ is not very large. This is due to the following facts:

The kinetic energy of a falling body and the piston at the initial instant $t=0$ is equal to $m_{1} v_{0}^{2} / 2$ (see (1.1) and (1.2)). This kinetic energy is taken up by the dissipated power and by the inertial forces power. The inertial forces power is defined by

$$
\rho \sum_{i=1}^{2}\left(\frac{\partial u_{i}}{\partial t}+u_{1} \frac{\partial u_{i}}{\partial r}+u_{2} \frac{\partial u_{i}}{\partial z}\right) u_{i} .
$$

Outside of the subdomain between the electrodes, the velocity of the fluid and the derivatives of the velocity are small (see Section 6). The density of the fluid $\rho$ is also small.

The module of the electric vector is maximal in between the electrodes, and it is almost zero outside of the subdomain between the electrodes (see Section 6). In between the electrodes, the velocity vector coincides with the direction of the axis $z$ and it is orthogonal to the electric vector (see Section 6). Therefore, the viscosity of the fluid in between the electrodes is greater by orders of magnitude than it is in the remaining part of the flow domain. The inertial forces in between the electrodes are negligible as compared with the internal forces caused by the viscous stresses.

Because of this, the predominant share of the kinetic energy of the falling body and the piston is taken up by the power dissipation in between the electrodes. In essence, the absorption of the kinetic energy of a falling body through the power dissipation is the predestination of the electrorheological shock absorber.

From the energy conservation law, it follows that neglect of the inertial forces increases the time it takes for the absorption of the kinetic energy of the falling body and the piston. Therefore, under neglect of the inertial forces, one obtains the upper bound of the time of absorption. Moreover, this upper bound is close to the time of absorption with regard for the inertial forces. 
The condition of incompressibility has the form:

$$
\operatorname{div} u^{t}=\frac{\partial u_{1}^{t}}{\partial r}+\frac{\partial u_{2}^{t}}{\partial z}+\frac{u_{1}^{t}}{r}=0 \text { in } \Omega_{a(t)} .
$$

In line with (2.32), the boundary conditions are the following:

$$
\begin{gathered}
\left.u_{1}^{t}\right|_{S_{a(t)}}=0,\left.u_{2}^{t}\right|_{S_{3 a(t)}}=v(t),\left.u_{2}^{t}\right|_{S_{4 a(t)}}=v(t) R_{1}^{2} R^{-2}, \\
\left.u_{2}^{t}\right|_{S_{a(t)} \backslash\left(\bigcup_{i=2}^{4} \bar{S}_{i a(t)}\right)}=0,\left.\frac{\partial u_{2}^{t}}{\partial r}\right|_{S_{2 a(t)}}=0 .
\end{gathered}
$$

Concerning the functions $\zeta$ and $\psi$ defining the viscosity (see (2.8)), we assume:

$(\mathbf{A 1}): \zeta:\left(y_{1}, y_{2}\right) \rightarrow \zeta\left(y_{1}, y_{2}\right)$ is a function continuous in $\mathbb{R}_{+} \times[0,1]$ and, in addition,

$$
0 \leq \zeta\left(y_{1}, y_{2}\right) \leq a_{0},\left(y_{1}, y_{2}\right) \in \mathbb{R}_{+} \times[0,1]
$$

where $a_{0}$ is a positive constant and $\mathbb{R}_{+}$is a set of nonnegative numbers.

(A2) $: \psi:\left(y_{1}, y_{2}, y_{3}\right) \rightarrow \psi\left(y_{1}, y_{2}, y_{3}\right)$ is a function continuous in $\mathbb{R}_{+}^{2} \times[0,1]$, and for an arbitrarily fixed $\left(y_{2}, y_{3}\right) \in \mathbb{R}_{+} \times[0,1]$, the partial function $\psi\left(., y_{2}, y_{3}\right): y_{1} \rightarrow \psi\left(y_{1}, y_{2}, y_{3}\right)$ is continuously differentiable in $\mathbb{R}_{+}$, and the following inequalities hold:

$$
\begin{gathered}
a_{2} \geq \psi\left(y_{1}, y_{2}, y_{3}\right) \geq a_{1}, \\
\psi\left(y_{1}, y_{2}, y_{3}\right)+2 \frac{\partial \psi}{\partial y_{1}}\left(y_{1}, y_{2}, y_{3}\right) y_{1} \geq a_{3}, \\
\left|\frac{\partial \psi}{\partial y_{1}}\left(y_{1}, y_{2}, y_{3}\right)\right| y_{1} \leq a_{4},
\end{gathered}
$$

where $a_{1}-a_{4}$ are positive constants.

Let us dwell on the physical sense of the inequalities (3.5)-(3.8). The inequalities (3.5) and (3.6) indicate that the viscosity is bounded from below and from above by positive constants. The inequality (3.7) implies that for fixed values of $|E|$, the derivative of the function $I(u) \rightarrow G(u)$ is positive, where $G(u)$ is the second invariant of the stress deviator

$$
G(u)=\sum_{i, j=1}^{n}\left(\sigma_{i j}(p, u, E)+p \delta_{i j}\right)^{2}=4[\varphi(I(u),|E|, \mu(u, E))]^{2} I(u) .
$$

This means that in case of simple shear flow, the shear stress increases with increasing shear rate. (3.8) is a restriction on $\frac{\partial \psi}{\partial y_{1}}$ for large values of $y_{1}$. The inequalities (3.5)-(3.8) are natural from a physical point of view.

The viscosity function is identified by approximation of a set of flow curves which are obtained experimentally by viscometric testing for different electric fields. The inequalities (3.5)-(3.8) are consistent with the shapes of the flow curves and enable one to approximate a set of flow curves over a wide range of shear rates with high degree of accuracy (see [14]).

At $\lambda=0$ the viscosity function $\varphi$ defined by $(2.8)$ is singular at $I(u)=0, \varphi(0,|E|, \mu(u, E))=\infty$, and the flow problems for such viscosity function reduce to the solution of variational inequalities (see [13]). The equation (2.8) with a small positive $\lambda$ defines a fluid with a finite but possibly large viscosity at $I(u)=0$. From a physical point of view, a fluid with bounded viscosity is more reasonable than a fluid with singular unbounded viscosity (in reality all viscosities are bounded). It is shown in [13] that the solutions of the problems with bounded viscosities converge to the solution of the problem with the singular viscosity as $\lambda$ tends to zero. Because of this, we assume that

$$
\lambda>0 \text {. }
$$

For a displacement of the piston $q \in[0, \mathcal{A}]$, we introduce the following sets:

$$
\begin{gathered}
\mathcal{H}_{0 q}=\left\{h\left|h=\left(h_{1}, h_{2}\right) \in C^{\infty}\left(\bar{\Omega}_{q}\right)^{2}, h_{1}\right|_{S_{q}}=0,\left.\frac{\partial h_{2}}{\partial r}\right|_{S_{2 q}}=0\right\}, \\
\mathcal{H}_{q}=\left\{h \mid h \in \mathcal{H}_{0 q}, h_{2}=0 \text { on } S_{3 q} \cup S_{4 q}\right\},
\end{gathered}
$$




$$
\mathcal{H}_{1 q}=\left\{h \mid h \in \mathcal{H}_{q}, \operatorname{div} h=0\right\} .
$$

We remind that $S_{2 q}$ is the set of the points $(r, z)$ of the boundary $S_{q}$ of $\Omega_{q}$ such that $r=0$, and $S_{3 q}$ and $S_{4 q}$ are the points of the boundary of the piston and the lower part of the boundary $S_{q}$.

Let $H_{q}$ and $H_{1 q}$ be the closures of $\mathcal{H}_{q}$ and $\mathcal{H}_{1 q}$ with respect to the norm

$$
\|h\|_{H_{q}}=\left(\int_{\Omega_{q}} I(h) r d r d z\right)^{\frac{1}{2}},
$$

where $I(h)$ is given by $(2.27)$.

Let also $H_{0 q}$ be the closure of $\mathcal{H}_{0 q}$ relative to the norm

$$
\|h\|_{H_{0 q}}=\left(\|h\|_{H_{q}}^{2}+\int_{S_{3 q}}|h|^{2} d s\right)^{\frac{1}{2}},
$$

where $d s=\left(d r^{2}+d z^{2}\right)^{\frac{1}{2}}$.

The norms (3.13) and (3.14) are equivalent to the norm of Sobolev space, see [20].

Let also $Y_{q}$ be the space of scalar functions which are square integrable in $\Omega_{q}$ with respect to the measure $r d r d z$. The norm in $Y_{q}$ is given by

$$
\|f\|_{Y_{q}}=\left(\int_{\Omega_{q}} f^{2} r d r d z\right)^{\frac{1}{2}}
$$

$Y_{q} / \mathbb{R}$ is the quotient space with the norm

$$
\|\dot{f}\|_{Y_{q} / \mathbb{R}}=\inf _{f \in \dot{f}}\|f\|_{Y_{q}} .
$$

We assume that the function of volume forces is given in each domain $\Omega_{q}$ so that

$$
K^{q} \in Y_{q}^{2}, q \in[0, \mathcal{A}]
$$

Everywhere below we use the following notation. If $U$ is a normed space, we denote the dual of $U$ by $U^{*}$, and by $(f, h)$ the duality between $U^{*}$ and $U$, where $f \in U^{*}$ and $h \in U$. The space $Y_{q}$ is identified with its dual space $Y_{q}^{*}$. The sign $\rightarrow$ denotes weak convergence in a Banach space.

\subsection{Weak solution of the problem}

Let $w^{t}$ be a function such that

$$
\begin{gathered}
w^{t} \in H_{0 a(t)},\left.w_{2}^{t}\right|_{S_{3 a(t)}}=v(t),\left.w_{2}^{t}\right|_{S_{4 a(t)}}=v(t) R_{1}^{2} R^{-2}, \\
\left.w_{2}^{t}\right|_{S_{a(t)} \backslash\left(\cup_{i=2}^{4} S_{i a(t)}\right)}=0, \operatorname{div} w^{t}=0 .
\end{gathered}
$$

For an arbitrary $t \in[0, T]$, we define the operators $L_{t}: H_{a(t)} \rightarrow H_{a(t)}^{*}$ and $B_{t}: \mathcal{L}\left(H_{a(t)}, Y_{a(t)}^{*}\right)$ as follows:

$$
\begin{gathered}
\left(L_{t}(y), h\right)=2 \int_{\Omega_{a(t)}} \varphi_{t} \epsilon_{i j}\left(w^{t}+y\right) \epsilon_{i j}(h) r d r d z, \quad y, h \in H_{a(t)}, \\
\left(B_{t} y, q\right)=\int_{\Omega_{a(t)}}(\operatorname{div} y) q r d r d z, \quad q \in Y_{a(t)} .
\end{gathered}
$$

The distance between the electrodes is small as compared to the length of the electrodes. So that, under the computation of the viscosity function, we can assume that in between the electrodes the vector of velocity relative to the electrodes is orthogonal to the vector of electric field strength, and the electric field strength is equal to zero in the remaining part of the domain under consideration.

The results of calculations presented in Section 6 and in [14] show that these assumptions are workable. 
With these assumptions the viscosity function $\varphi_{t}$ in (3.19) takes the following form:

$$
\varphi_{t}=\left\{\begin{array}{l}
\zeta\left(\left|E^{t}\right|, 0\right)\left(\lambda+I\left(w^{t}+y\right)\right)^{-\frac{1}{2}}+\psi\left(I\left(w^{t}+y\right),\left|E^{t}\right|, 0\right) \quad \text { in } \mathcal{X}_{a(t)}, \\
\zeta(0,0)\left(\lambda+I\left(w^{t}+y\right)\right)^{-\frac{1}{2}}+\psi\left(I\left(w^{t}+y\right), 0,0\right) \quad \text { in } \Omega_{a(t)} \backslash \mathcal{X}_{a(t)},
\end{array}\right.
$$

where $\mathcal{X}_{a(t)}$ is the domain between the electrodes (see Figure 2),

$$
\mathcal{X}_{a(t)}=\left\{(r, z) \mid r \in\left(r_{1}, r_{2}\right), z \in\left(l-l_{1}(0)-a(t)-d, l-l_{1}(0)-a(t)\right)\right\} .
$$

We consider the following problem: Find a pair of functions $\left(y^{t}, p^{t}\right)$ satisfying

$$
\begin{gathered}
y^{t} \in H_{a(t)}, \quad p^{t} \in Y_{a(t)} / \mathbb{R}, \\
\left(L_{t}\left(y^{t}\right), h\right)-\left(B_{t}^{*} p^{t}, h\right)=\left(K^{a(t)}, h\right), \quad h \in H_{a(t)}, \\
\left(B_{t} y^{t}, q\right)=0, \quad q \in Y_{a(t)} .
\end{gathered}
$$

The pair $\left(u^{t}=w^{t}+y^{t}, p^{t}\right)$, where $\left(y^{t}, p^{t}\right)$ is the solution of the problem (3.21)-(3.23), will be called the weak solution of the problem (3.1)-(3.4).

The next theorem follows from the results of [13] and [18].

Theorem 3.1 Suppose that $a(t)$ and $E(t)$ are given, where $t \in[0, T]$, and the conditions $(A 1),(A 2)$, (3.9), (3.17) and (3.18) are satisfied. Then there exists a unique solution to the problem (3.21)-(3.23).

The function $p^{t}$ is defined with an accuracy of a constant addend. The pressure in the shock absorber depends on the pressure in the gas reservoir (see Figure 1). The pressure in the reservoir should be sufficiently large in order that the normal component of the force acting on the top lid of the shock absorber to be positive, i.e. the normal component of the force acting on the fluid at the cross-section $z=l$ (see Figure 2) to be negative. Otherwise, the upper chamber (chamber A in Figure 1) is bound to be not fully filled with the fluid, when the piston moves downwards. In such an event the cavitation appears, but it is not admissible.

Let

$$
S_{0}=\left\{(r, z) \mid z=l, r \in\left(R_{1}, R\right)\right\} .
$$

The normal component of the surface force acting on the fluid at the cross-section $z=l$ at an instant $t$ is given by

$$
F_{2}(t)=\left.\left(-p^{t}+2 \varphi_{t} \frac{\partial\left(w_{2}^{t}+y_{2}^{t}\right)}{\partial z}\right)\right|_{S_{0}} .
$$

Taking into account (3.17) and the results of [2], Section 18, it can be shown that

$$
F_{2}(t) \in H^{-\frac{1}{2}}\left(S_{0}\right), \quad t \in[0, T] .
$$

In order that the upper chamber (chamber A in Figure 1) be fully filled with the fluid, the distribution $F_{2}(t)$ must be nonpositive. In other words, it should be so that $\left(F_{2}(t), \beta\right) \leq 0$ for all $t$ and an arbitrary $\beta$ such that $\beta \in H_{0}^{1}\left(S_{0}\right), \beta(s) \geq 0$ at $s \in S_{0}$.

Let $\delta$ be a small positive constant and $\alpha$ be a function such that

$$
\begin{gathered}
\alpha \in C^{1}\left(\left[R_{1}, R\right]\right), \alpha(r) \geq 0 \text { at } r \in\left[R_{1}, R\right], \\
\alpha(r)=1 \text { at } r \in\left[R_{1}+\delta, R-\delta\right], \alpha\left(R_{1}\right)=0, \alpha(R)=0 .
\end{gathered}
$$

Then $\alpha \in H_{0}^{1}\left(S_{0}\right)$ and we take the constant addend of $p^{t} \in Y_{t} / \mathbb{R}$ so that

$$
\left(F_{2}(t), \alpha\right)=-\mathcal{C}, \quad t \in[0, T]
$$

where $\mathcal{C}$ is a positive constant.

In this case the function $p^{t}$ is defined uniquely. The condition (3.28) denotes that the total axial force 
acting on the top lid of the shock absorber is positive. We assume that (3.28) is the condition of without cavitation flow. Provided that the pressure in the gas reservoir is so that $-\left(F_{2}(t), \alpha\right) \geq \mathcal{C}$, the fluid flows without cavitation.

The normal component of the surface force acting on the fluid at the cross-section $z=-b(t)$, i.e. on $S_{4 a(t)}$ is defined by

$$
F_{3}(t)=\left.\left(p^{t}-2 \varphi_{t} \frac{\partial\left(w_{2}^{t}+y_{2}^{t}\right)}{\partial z}\right)\right|_{S_{4} a(t)}
$$

Since $F_{3}(t) \in H^{-\frac{1}{2}}\left(S_{4 a(t)}\right)$ for all $t \in[0, T]$, we can consider that the total axial force acting on the fluid on the boundary $S_{4 a(t)}$ is equal to $\left(F_{3}(t), \alpha_{1}\right)$, where

$$
\alpha_{1} \in C^{1}([0, R]), \alpha_{1}(r) \geq 0, \alpha_{1}(r)=1 \text { at } r \in[0, R-\delta], \alpha_{1}(R)=0 .
$$

Therefore, if the pressure in the gas reservoir $p_{g}$ is so that

$$
p_{g} \geq \frac{\left(F_{3}(t), \alpha\right)}{\pi R^{2}}
$$

then the fluid flows without cavitation.

We define the axial component of the total force acting on the piston (the drag) at an instant $t$ as follows (compare with $(2.34))$ :

$$
G(t, v(t), V(t))=-\int_{S_{3 a(t)}}\left[2 \varphi_{t} \epsilon_{31}\left(w^{t}+y^{t}\right) \nu_{1}+\left(-p^{t}+2 \varphi_{t} \epsilon_{33}\left(w^{t}+y^{t}\right)\right) \nu_{2}\right] \alpha_{4}(t) d s
$$

Here $\varphi_{t}$ is defined by (3.20), and $\alpha_{4}(t)$ is a nonnegative smooth function that is equal to unit everywhere on $S_{3 a(t)}$ with the exception of small vicinities of the points $s_{1}=\left(R_{1}, l\right), s_{2}=\left(R, l-l_{1}(t)\right), s_{3}=$ $\left(R, l-l_{1}(t)-d\right)$, in addition, $\alpha_{4}\left(s_{i}\right)=0, i=1,2,3$.

The function $\alpha_{4}(t)$ is introduced in (3.32) since the function of surface forces acting on the piston is an element of the space $H^{-\frac{1}{2}}\left(S_{3 a(t)}\right)$.

Thus, the problem on electrorheological shock absorber consists in finding the functions $v(t), E^{t}=$ $-\operatorname{grad}\left(\theta_{0 t}+\theta_{1 t}\right), u^{t}=w^{t}+y^{t}$, and $p^{t}$ such that the equations (1.1), (1.2), (2.25), (3.21)-(3.23), (3.28) and (3.32) are satisfied.

The domain $\Omega_{a(t)}$ is unknown, and it is defined by $a(t)$ (see (2.35) and Figure 2). In this case the values $l_{1}(0), v(0)=v_{0}$, and $m_{1}$ are given.

\section{Numerical solution of the problem on shock absorber}

We consider the method of semi-discretization of the problem. Let us choose a positive integer $N$, let $k$ denote the corresponding time-step: $k=T / N$ and $t_{n}$ be the subdivisions of $[0, T]: t_{n}=n k, 0 \leq n \leq N$.

We denote the approximations of the functions $v, a, E, u, y$ and $p$ by $v^{k}, a^{k}, E^{k}, u^{k}, y^{k}$, and $p^{k}$, respectively. The approximations of these functions at an instant $t_{n}$ are denoted by $v^{k n}, a^{k n}, E^{k n}, u^{k n}, y^{k n}$, and $p^{k n}$, respectively.

Initial step, $n=0, t=0$

- Compute $\theta_{10}$, the solution of the problem (2.25) at $t=0$ in the domain $\Omega_{0}$. The electric field at $t=0$ is determined by the following expression:

$$
E^{0}=-\operatorname{grad}\left(\theta_{00}+\theta_{10}\right)
$$

- Find $y^{0}$ and $p^{0}$ the solution of the problem (3.21)-(3.23) and (3.28) at $t=0$. 
- Compute $G(0, v(0), V(0))$ and then $\frac{d v}{d t}(0)$ by using (3.32) and (1.1),

$$
\frac{d v}{d t}(0)=\frac{1}{m_{1}} G(0, v(0), V(0)) \text {. }
$$

- Set $v^{k 0}=v(0)=v_{0}, E^{k 0}=E^{0}, y^{k 0}=y^{0}, u^{k 0}=w^{0}+y^{0}, p^{k 0}=p^{0}$, and

$$
\begin{gathered}
v^{k}(t)=v_{0}+\frac{d v}{d t}(0) t, \quad t \in[0, k] \\
a^{k 1}=-\int_{0}^{k}\left(v_{0}+\frac{d v}{d t}(0) t\right) d t=-v_{0} k-\frac{k^{2}}{2} \frac{d v}{d t}(0), \\
v^{k 1}=v_{0}+\frac{d v}{d t}(0) k .
\end{gathered}
$$

We mention here that the minus sign is taken in (4.4) since the displacement is positive by definition, while the velocity is negative.

Now, let $v^{k n}, a^{k n}, E^{k(n-1)}, y^{k(n-1)}, p^{k(n-1)}$ and $u^{k(n-1)}=w^{t_{n-1}}+y^{k(n-1)}$ be computed.

Time step $t=t_{n}, 1 \leq n \leq N$.

- Compute $\theta_{1 t_{n}}$ the solution of the problem (2.25) for $t=t_{n}$ in the domain $\Omega_{a^{k n}}$. Then, compute $E^{t_{n}}$ by

and set $E^{k n}=E^{t_{n}}$.

$$
E^{t_{n}}=-\operatorname{grad}\left(\theta_{0 t_{n}}+\theta_{1 t_{n}}\right)
$$

- Compute $y^{t_{n}}$ and $p^{t_{n}}$ the solution of the problem (3.21)-(3.23), and (3.28) for $t=t_{n}$ in the domain $\Omega_{a^{k n}}$. In this case the function $w^{t_{n}}$ in the operator $L_{t_{n}}$ satisfies the conditions (3.18) with $v\left(t_{n}\right)$ replaced by $v^{k n}$.

Set $y^{k n}=y^{t_{n}}, p^{k n}=p^{t_{n}}, u^{k n}=w^{t_{n}}+y^{k n}$.

- Compute $G\left(t_{n}, v^{k n}, V\left(t_{n}\right)\right)$ by $(3.32)$, where $S_{3 a\left(t_{n}\right)}$ is replaced by $S_{3 a^{k n}}$, and take

$$
\frac{d v^{k}}{d t}\left(t_{n}\right)=\frac{1}{m_{1}} G\left(t_{n}, v^{k n}, V\left(t_{n}\right)\right) \text {. }
$$

- Set

$$
\begin{gathered}
v^{k}(t)=v^{k n}+\frac{d v^{k}}{d t}\left(t_{n}\right)\left(t-t_{n}\right), \quad t \in\left[t_{n}, t_{n+1}\right], \\
v^{k(n+1)}=v^{k n}+\frac{d v^{k}}{d t}\left(t_{n}\right) k \\
a^{k}(t)=a^{k n}-\int_{t_{n}}^{t} v^{k}(\tau) d \tau=a^{k n}-v^{k n}\left(t-t_{n}\right)-\frac{1}{2} \frac{d v^{k}}{d t}\left(t_{n}\right)\left(t-t_{n}\right)^{2}, \quad t \in\left[t_{n}, t_{n+1}\right], \\
a^{k(n+1)}=a^{k n}-v^{k n} k-\frac{1}{2} \frac{d v^{k}}{d t}\left(t_{n}\right) k^{2} .
\end{gathered}
$$

We define the values of the functions $E^{k}, u^{k}$ and $p^{k}$ in the intervals $n k<t<(n+1) k$ as follows:

$$
\begin{gathered}
E^{k}(t)=E^{k n}+\frac{t-n k}{k}\left(E^{k(n+1)}-E^{k n}\right), \\
u^{k}(t)=u^{k n}+\frac{t-n k}{k}\left(u^{k(n+1)}-u^{k n}\right), \\
p^{k}(t)=p^{k n}+\frac{t-n k}{k}\left(p^{k(n+1)}-p^{k n}\right),
\end{gathered}
$$

for $0 \leq n \leq N-1$

\section{On the convergence of approximations}

Below we use the ideas of the works by Litvinov [19], Section 6.3 and [21].

Let $q$ be a displacement of the piston, $q \in[0, \mathcal{A}]$. We partition the domain $\Omega_{q}$ into three parts, $\Omega_{0 q}, \Omega_{1 q}$, 
and $\Omega_{2 q}$,

$$
\begin{aligned}
& \Omega_{0 q}=\left\{(r, z) \mid r \in(0, R), z \in\left(-q R_{1}^{2} R^{-2}, l-l_{1}(0)-q-d\right]\right\}, \\
& \Omega_{1 q}=\left\{(r, z) \mid r \in\left(r_{1}, r_{2}\right), z \in\left(l-l_{1}(0)-q-d, l-l_{1}(0)-q\right]\right\}, \\
& \Omega_{2 q}=\left\{(r, z) \mid r \in\left(R_{1}, R\right), z \in\left(l-l_{1}(0)-q, l\right)\right\} .
\end{aligned}
$$

Clearly, $\Omega_{q}=\Omega_{0 q} \cup \Omega_{1 q} \cup \Omega_{2 q}$. We denote the points of $\Omega_{q}$ at $q>0$ by $\left(r_{q}, z_{q}\right)$.

We define a mapping $\mathcal{P}_{q}: \bar{\Omega}_{0} \rightarrow \bar{\Omega}_{q}$ as follows: $(r, z) \in \bar{\Omega}_{0}, \mathcal{P}_{q}(r, z)=\left(r_{q}, z_{q}\right) \in \bar{\Omega}_{q}$,

$$
\begin{gathered}
r_{q}=r, z_{q}=\frac{z\left[l-l_{1}(0)-q\left(1-R_{1}^{2} R^{-2}\right)-d\right]}{l-l_{1}(0)-d}-q R_{1}^{2} R^{-2} \quad \text { at }(r, z) \in \bar{\Omega}_{0 q}, \\
r_{q}=r, z_{q}=z-q \text { at }(r, z) \in \bar{\Omega}_{1 q}, \\
r_{q}=r, z_{q}=l-\frac{\left(l_{1}(0)+q\right)(l-z)}{l_{1}(0)} \quad \text { at }(r, z) \in \bar{\Omega}_{2 q} .
\end{gathered}
$$

The function $\mathcal{P}_{q}$ defines a one-to-one mapping of $\bar{\Omega}_{0}$ onto $\bar{\Omega}_{q}$, moreover it is continuously differentiable almost everywhere in $\bar{\Omega}_{0}$. The function $\mathcal{P}_{q}$ is continuous in $\bar{\Omega}_{0}$, moreover it is continuously differentiable almost everywhere in $\bar{\Omega}_{0}$. The function $\mathcal{P}_{q}$ is not differentiable at points $(r, z)$ such that $z=l-l_{1}(0)-d$, $r \in\left[r_{1}, r_{2}\right]$, and $z=l-l_{1}(0), \quad r \in\left[r_{1}, r_{2}\right]$.

We suppose that

$$
V \in C([0, T]), \quad \text { the function } q \rightarrow K^{q} \circ \mathcal{P}_{q} \text { belongs to } C\left([0, \mathcal{A}] ; Y_{0}^{2}\right),
$$

see $(3.17)$.

We denote the functions $t \rightarrow E^{t} \circ \mathcal{P}_{a(t)}, t \rightarrow u^{t} \circ \mathcal{P}_{a(t)}$ and $t \rightarrow p^{t} \circ \mathcal{P}_{a(t)}$ by $E \circ \mathcal{P}_{a}, u \circ \mathcal{P}_{a}$, and $p \circ \mathcal{P}_{a}$, respectively. Similarly, we denote the functions $t \rightarrow E^{k}(t) \circ \mathcal{P}_{a^{k}(t)}, t \rightarrow u^{k}(t) \circ \mathcal{P}_{a^{k}(t)}$ and $t \rightarrow p^{k}(t) \circ \mathcal{P}_{a^{k}(t)}$ by $E^{k} \circ \mathcal{P}_{a^{k}}, u^{k} \circ \mathcal{P}_{a^{k}}$ and $p^{k} \circ \mathcal{P}_{a^{k}}$, respectively.

Theorem 5.1 Suppose that the conditions (A1), (A2), (2.22), (2.23), (3.9), (3.17) and (3.18) are satisfied. Let also (5.3) hold and $a(T) \leq \mathcal{A}$. Then there exists a unique solution $v, a, E, u, p\left(E^{t}=\right.$ $\left.-\operatorname{grad}\left(\theta_{0 t}+\theta_{1 t}\right), u^{t}=w^{t}+y^{t}\right)$ of the coupled problem (1.1), (1.2), (2.25), (2.35), (3.21)-(3.23), (3.28) and (3.32) such that

$$
\begin{gathered}
v \in C^{1}([0, T]), \quad a \in C^{2}([0, T]), \\
E \circ \mathcal{P}_{a} \in C\left([0, T] ; Y_{0}^{2}\right), \quad u \circ \mathcal{P}_{a} \in C\left([0, T] ; H_{00}\right), \quad p \circ \mathcal{P}_{a} \in C\left([0, T] ; Y_{0}\right) .
\end{gathered}
$$

Moreover,

$$
\begin{array}{cc}
E^{k} \circ \mathcal{P}_{a^{k}} \rightarrow E \circ \mathcal{P}_{a} \quad \text { in } C\left([0, T] ; Y_{0}^{2}\right), \\
u^{k} \circ \mathcal{P}_{a^{k}} \rightarrow u \circ \mathcal{P}_{a} \quad \text { in } C\left([0, T] ; H_{00}\right), \\
p^{k} \circ \mathcal{P}_{a^{k}} \rightarrow p \circ \mathcal{P}_{a} \quad \text { in } C\left([0, T] ; Y_{0}\right),
\end{array}
$$

as $k \rightarrow 0$.

It should be pointed out that in the case when $a\left(t_{1}\right)=\mathcal{A}$, where $t_{1}<T$, the problem under consideration can be studied on the interval $\left[0, t_{1}\right]$.

Proof of Theorem 5.1 The existence of a unique solution $E^{k}, u^{k}, p^{k}, v^{k}, a^{k}$ of the semi-discrete problem follows from the theorems 2.1 and 3.1. Let $k \rightarrow 0$.

By virtue of (3.5)-(3.8), and (3.28) $G\left(t, v^{k}(t), V(t)\right)>0$ for all $t \in[0, T]$ and all $k$. So that (1.1) implies $\frac{d v^{k}}{d t}(t)>0$, and (1.2) yields

$$
\left|v^{k}(t)\right|<\left|v_{0}\right|, \quad t \in[0, T] .
$$


It follows from (5.9) and from the results of [19], Section 6.3, that

$$
\begin{gathered}
\left\|u^{k}(t) \circ \mathcal{P}_{a^{k}(t)}\right\|_{H_{00}} \leq C_{1}, \\
\left\|p^{k}(t) \circ \mathcal{P}_{a^{k}(t)}\right\|_{Y_{0}} \leq C_{2}
\end{gathered}
$$

for all $k$ and $t \in[0, T]$, in addition,

$$
\begin{gathered}
\left|G\left(t, v^{k}(t), V(t)\right)\right| \leq C_{3}, \\
\left|\frac{d v^{k}}{d t}(t)\right| \leq C_{4} .
\end{gathered}
$$

By virtue of (5.9) and (5.13), a subsequence $\left\{v^{m}\right\}$ can be extracted such that

$$
v^{m} \rightarrow \tilde{v} \quad \text { in } C([0, T]) .
$$

Let

and

$$
\tilde{a}(t)=-\int_{0}^{t} \tilde{v}(\tau) d \tau
$$

$$
\tilde{E}(t)=-\operatorname{grad}\left(\theta_{0 t}+\theta_{1 t}\right),
$$

where $\theta_{1 t}$ is the solution of the problem (2.25) in the domain $\Omega_{\tilde{a}(t)}, \theta_{0 t}$ satisfies $(2.22)$ with $a(t)$ being replaced by $\tilde{a}(t)$.

Let also $\tilde{u}(t)=w^{t}+y^{t}, \tilde{p}(t)=p^{t}$, where $y^{t}$ and $p^{t}$ is the solution of the problem (3.21)-(3.23), (3.28) in the domain $\Omega_{\tilde{a}(t)}, w^{t}$ satisfies (3.18), where $a(t)$ is replaced by $\tilde{a}(t)$ and $v(t)$ is replaced by $\tilde{v}(t)$.

The relation (5.14) and the results of [19], Section 6.3 imply the following:

$$
\begin{array}{cc}
E^{m} \circ \mathcal{P}_{a^{m}} \rightarrow \tilde{E} \circ \mathcal{P}_{\tilde{a}} \quad \text { in } C\left([0, T] ; Y_{0}^{2}\right), \\
u^{m} \circ \mathcal{P}_{a^{m}} \rightarrow \tilde{u} \circ \mathcal{P}_{\tilde{a}} \quad \text { in } C\left([0, T] ; H_{00}\right), \\
p^{m} \circ \mathcal{P}_{a^{m}} \rightarrow \tilde{p} \circ \mathcal{P}_{\tilde{a}} \quad \text { in } C\left([0, T] ; Y_{0}\right),
\end{array}
$$

and the functions $v=\tilde{v}, a=\tilde{a}, E=\tilde{E}, u=\tilde{u}, p=\tilde{p}$ is the solution of our coupled problem (1.1), (1.2), (2.25), (3.21)-(3.23), (3.28), (3.32). In this case $E^{t}=-\operatorname{grad}\left(\theta_{0 t}+\theta_{1 t}\right)$ and $u^{t}=w^{t}+y^{t}$.

It follows from (5.14) that $v \in C([0, T])$. By applying the results of [19], Section 6.3, we obtain that $t \rightarrow G(t, v(t), V(t))$ is a function which is continuous on $[0, T]$, and so $(1.1)$ implies that $v \in C^{1}([0, T])$.

The function $v$ takes negative values (see (1.2)) and is increasing. Since the viscosity function is bounded (see (3.5), (3.6), and (3.9)), there exists a constant $k>0$ such that

$$
G(t, v(t), V(t)) \leq k|v(t)|, \quad t \in[0, T] .
$$

(1.1) and (5.20) yield

$$
v_{0} \exp \left(-\frac{k}{m_{1}} t\right) \geq v(t), \quad t \in[0, T]
$$

Therefore,

$$
v(t)<0 \quad \text { at } t \in[0, T], \quad T<\infty .
$$

Since $v \in C^{1}([0, T])$ and (5.22) is satisfied, an arbitrary solution of the coupled problem can be approximated by the solution of the semi-discretized problem considered in Section 4. The solution of the semi-discretized problem is unique and so, the solution of the coupled problem is also unique. Now (5.17)-(5.19) yield (5.6)-(5.8).

Remark [Possible generalization] Problems on shock absorber can be investigated with regard for the inertial forces in the motion equations for the fluid. In addition, one can consider that the domain of flow is not axially symmetric. 


\section{Simulation results}

For the simulation, we consider the Rheobay TP AI 3656 Electrorheological fluid, a product of Rheobay $[1,14]$. The experimentally obtained flow curves (relating the shear stress to the shear rate) of this product, corresponding to different electric field strengths orthogonal to the velocity fields, have been approximated by cubic splines. The viscosity function was then calculated from these splines. see [14] for details.

The calculations were fulfilled for the following data set, cf. Figure $2, R=0.023$ meter, $R_{1}=0.005$ meter, $r_{1}=0.013$ meter, $r_{2}=0.017$ meter, $l=0.14$ meter, $l_{1}(0)=0.02$ meter, $d=0.04$ meter, and $\mathcal{C}=0.5$ Newton (cf. (3.28)) and $m_{1}=50 \mathrm{~kg}$., $K=\left(K_{1}, K_{2}\right)=0$.

The results of calculations are presented in figures $3-7$.

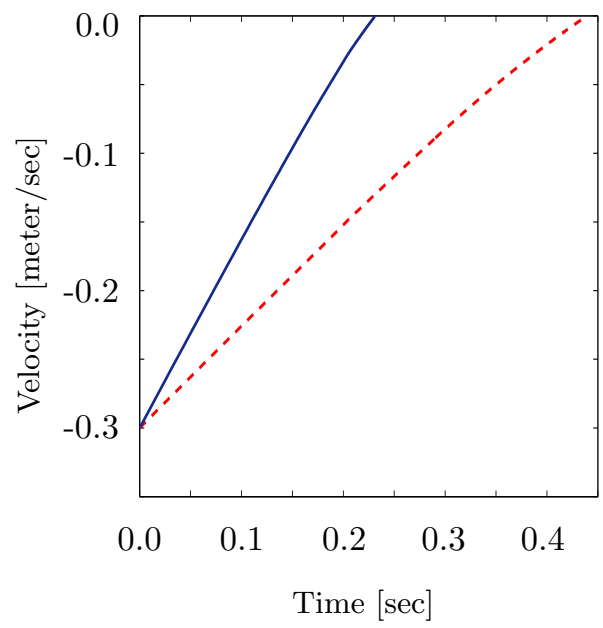

Figure 3. Velocity of the piston as a function of time. The curves correspond to the voltages $V=9 \times 10^{3}$ Volt (solid line) and $V=6 \times 10^{3}$ Volt (dashed line).

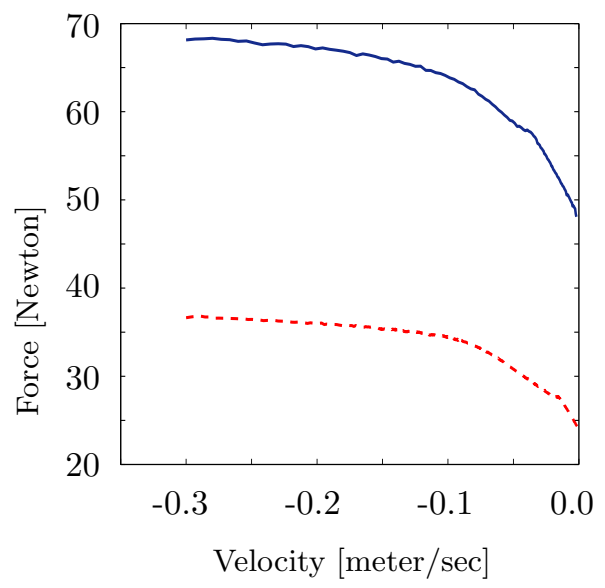

Figure 4. Drag force acting on the piston plotted against the velocity. The curves correspond to the two cases considered in the Figure 3. In the case that $\lambda=0$ in (2.8) and in (3.20), respectively, the piston comes into motion if and only if the total force acting on it attains some finite value, that depends on the voltage on the electrodes. The near phenomenon occurs at small positive $\lambda$. The drag force shown here is relatively large at very small velocity of the piston. 

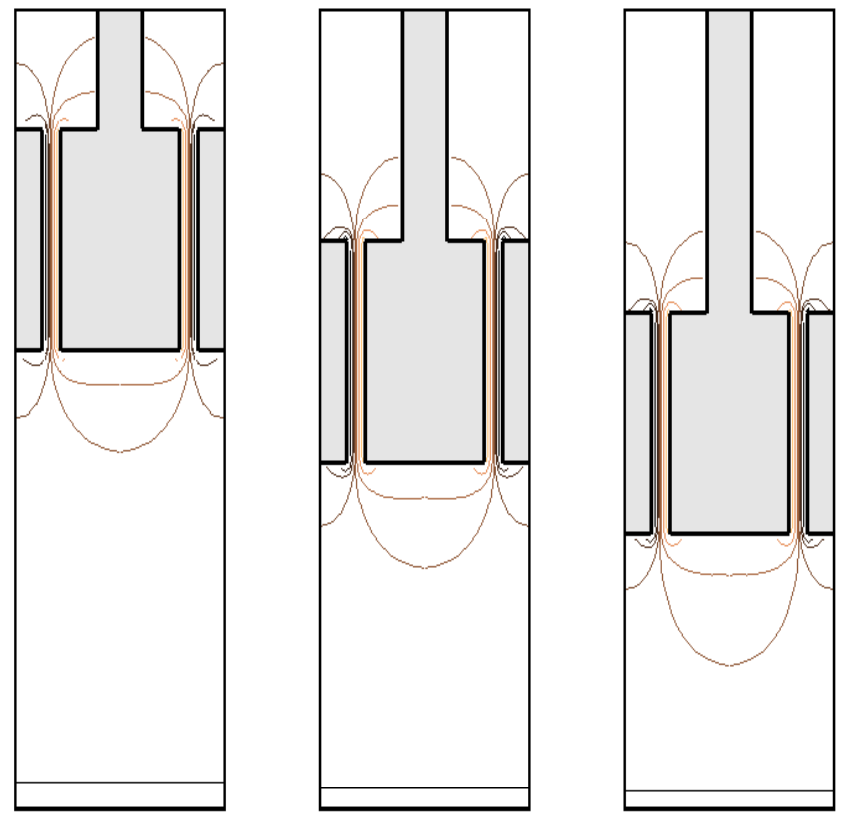

Figure 5. Contour plots for the electric potential at $V=9 \times 10^{3}$ Volt at time $t=0.006,0.090,0.186$ second.

\section{Conclusion}

1. The drag force acting on the piston depends on the voltage, on the velocity of the piston and on its position (on $a(t)$ ), and on the pressure in the gas reservoir. The pressure in the gas reservoir should be sufficiently large, so as the condition (3.31) to be satisfied. Otherwise, the chamber A (see Figure 1) is bound to be not fully filled with the fluid and the cavitation appears.

2. The impact force, i.e. the drag force is large at high voltage, even though the module of velocity of the piston is very small (see Figure 4).

3. In between the electrodes, the electric vector is essentially directed along the direction of the axis $r$ or in the opposite direction, depending on the sign of $V(t)$; practically $E_{1}(t)=V(t) /\left(r_{2}-r_{1}\right), E_{2}(t)=0$ in between the electrodes. The electric field falls drastically as the distance to the electrodes increases, see Figure 5. One can consider that electric vector equals zero outside of the domain between the electrodes.

4. In between the electrodes, the direction of the vector of velocity of the fluid coincides essentially with the direction of the axis $z$, and the velocity vector is orthogonal to the electric vector.

5. Profile of the relative velocity of the fluid in between the electrodes tends to the rectangular profile as the voltage increases, at zero voltage the profile is close to the corresponding profile of the Newtonian fluid, see Figure 7.

6. The module of the velocity of the piston can be brought to an arbitrarily small value by the electrorheological fluid. However, the fluid cannot reduce it to zero in any finite time, since the viscosity of the fluid takes finite values (see (5.22)). When the electrorheological shock absorber operates, an additional force, that is created by a spring, reduces the velocity of the piston to zero, then restores the piston to its original position. 


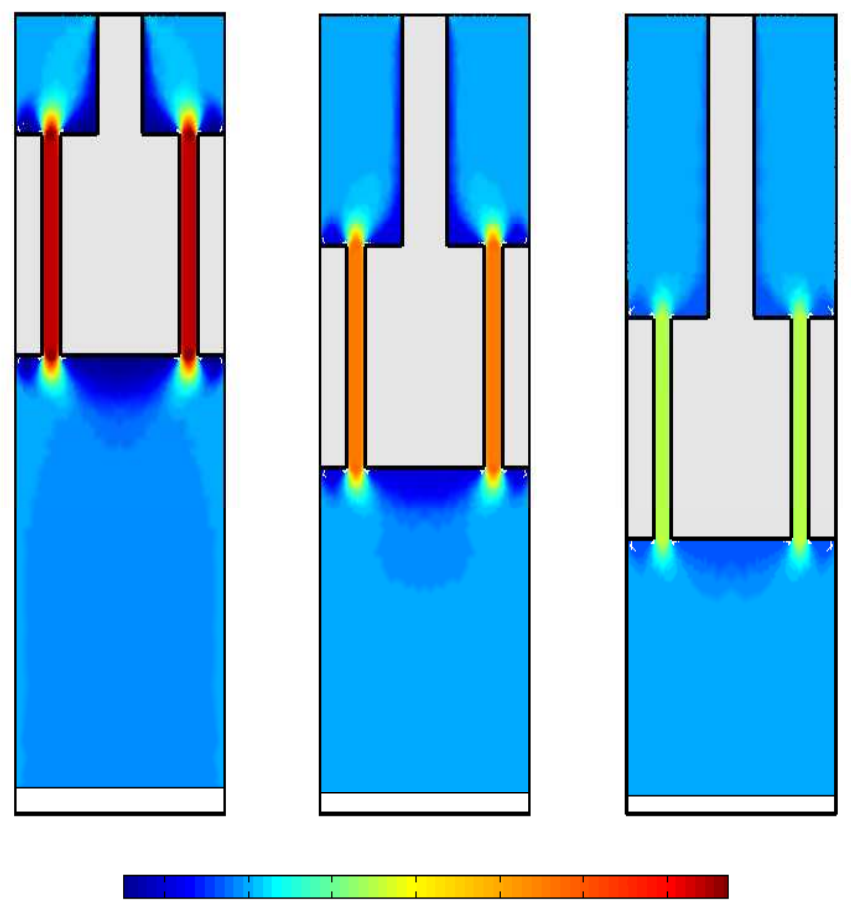

$\begin{array}{lllllllll}-0.2 & 0.0 & 0.2 & 0.4 & 0.6 & 0.8 & 1.0 & 1.2 & {[\mathrm{~meter} / \mathrm{sec}]}\end{array}$

FigurE 6. Surface plots of the longitudinal component $u_{2}$ of the velocity of the fluid for $V=9 \times 10^{3}$ Volt at time $t=0.006,0.090,0.186$ second.

\section{Acknowledgement}

The work of the authors has been supported by the German National Science Foundation (DFG) within the Collaborative Research Center SFB 438.

\section{References}

[1] BAYer Provisional Product Information of Rheobay TP AI 3565 and Rheobay TP AI 3566. Bayer Silicones Rheobay, Bayer.

[2] Baiocchi, C., \& CApelo, A. 1984 Variational and Quasivariational Inequalities. Applications to Free Boundary Problems. John Wiley and Sons.

[3] Belonosov, M.S. \& Litvinov, W.G. 1996 Finite element method for nonlinearly viscous fluids. Zeitschrift für Angewandte Mathematik und Mechanik 76, 307-320.

[4] Block, H. \& Kelly, J.P. 1988 Electro-rheology. Journal of Physics D: Applied Physics 21, 1661-1677.

[5] Bossis, G. (Ed.) 2002 Electrorheological Fluids and Magnetorheological Suspensions. Proceedings of the Eight International Conference, Nice, France 9-13 July 2001. World Scientific.

[6] Ellam, D.J., Atkin, R.J. \& Bullough, W.A. 2005 Analysis of a smart clutch with cooling flow using two-dimensional Bingham plastic analysis and computational fluid dynamics. Proceedings of the institution of Mechanical Engineers, Part A: Journal of Power and Energ 219, 639-652, 2005.

[7] Ellam, D.J., Bullough, W.A. \& Atkin, R.J. 2005 Modelling the flow of an electrostructured fluid in transient operation. Proceedings of the institution of Mechanical Engineers, Part A: Journal of Power and Energy 219, 61-76.

[8] Filisko, F. 1995 Overview of ER technology. Progress in ER Technology, Havelka K. (Ed.). New York, Plenum Press. 


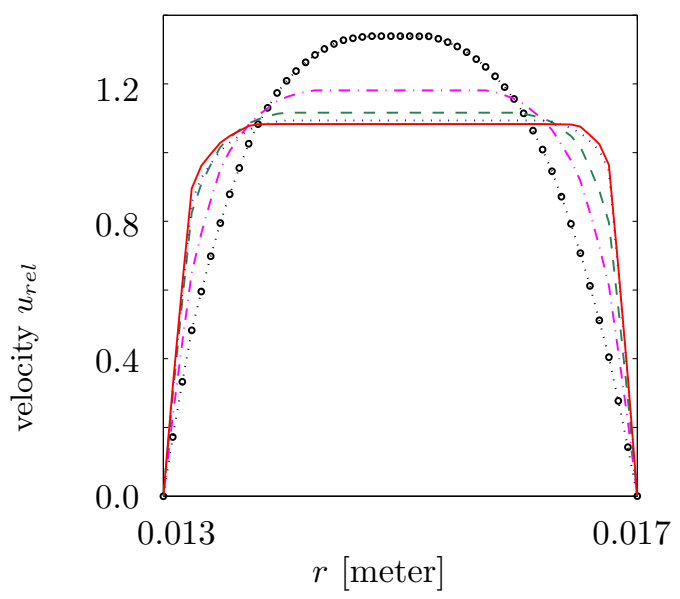

Figure 7 . Profiles of the dimensionless relative velocity of the fluid $u_{r e l}$ in between the electrodes, $u_{r e l}=$ $\left(u_{2}-v\right) \Upsilon^{-1}$, where $\Upsilon$ is the flow rate relative to the electrodes, $\Upsilon=\left(\int_{r_{1}}^{r_{2}} r d r\right)^{-1} \int_{r_{1}}^{r_{2}} r\left(u_{2}-v\right)\left(r, z_{1}\right) d r$. Here $z_{1}=l-l_{1}(t)-\frac{d}{2}, t \in(0, T), u_{r e l}$ is practically independent of $t$. The curves correspond to the following voltages: $V=0$ Volt (dotted-circled line), $1 \times 10^{3}$ Volt (dashed-dotted line), $3 \times 10^{3}$ Volt (dashed line), $6 \times 10^{3}$ Volt (dotted line) and $9 \times 10^{3}$ Volt (solid line).

[9] Fư̌IK S., Krtochvil, A. \& NeČAs, J. 1973 Kachanov-Galerkin method. Commentationes Mathematicae Universitatis Carolinae 14, 651-659.

[10] Gajewski, H., Gröger, K., \& Zacharias 1974 Nichtlineare Operatorgleichungen und Operatordifferentialgleichungen. Akademie-Verlag, Berlin.

[11] Girault, V. \& Raviart, P. 1986 Finite Element Approximation of the Navier-Stokes Equations. SpringerVerlag, Berlin.

[12] Güntner, M. \& Prokert, G. 1997 Existence for the quasistationary motion of a free capillary liquid drop. Zeitschrift für Analysis und ihre Anwendungen 16, 311-348.

[13] Hoppe, R.H.W. \& Litvinov, W.G. 2004 Problems on electrorheological fluid flows. Communication in Pure and Applied Analysis 3, no. 4, 809-848.

[14] Hoppe, R.H.W., Litvinov, W.G. \& Rahman T. 2005 Problems of stationary flow of electrorheological fluids in the cylindrical coordinate system. SIAM Journal of Applied Mathematics 65, No. 5, 1633-1656.

[15] Ladyzhenskaya, O. A.\& Solonnikov, V.A. 1976 Some problems of vector analysis and generalized formulation of boundary value problems for the Navier-Stokes equations. Zap. Nauchn. Sem. Leningrad. Otdel Math. Inst. Steklov (LOMI) 59, 81-116 (in Russian).

[16] Landau, L.D. \& Lifshitz E.M. 1984 Electrodynamics of Continuous Media. Pergamon, Oxford.

[17] Lions, J.-L. 1969 Quelques Méthodes de Résolution des Problèmes aux Limites non Linéaires. Dunod Gauthier-Villars, Paris.

[18] Litvinov, W.G. 1982 Motion of Nonlinearly Viscous Fluid. Moscow, Nauka (in Russian).

[19] Litvinov, W.G. 2000 Optimization in Elliptic Problems with Applications to Mechanics of Deformable Bodies and Fluid Mechanics. Birkhäuser.

[20] Litvinov, W.G. 1996 A problems on non steady flow of a nonlinear viscous fluid in a deformable pipe. Methods of Functional Analysis and Topology 2, No 3-4, 85-113.

[21] Litvinov, W.G. 2003 A model and a general problem on plastic flow under large deformations. Zeitschrift für Angewandte Mathematik und Mechanik 83, 291-310.

[22] Parthasarathy, M. \& Kleingenberg, D.J. 1996 Electrorheology: mechanisms, and models. Material Science and Engineering R17, 57-103.

[23] Pukhnachov, V.V. 1989 Motion of Viscous Fluid with Free Boundaries. Novosibirsk University Publication, Novosibirsk (in Russian).

[24] Pukhnachov, V.V. 1998 The quasistationary approximation in the problem of the motion of an isolated volume of a viscous incompressible capillary liquid. Journal of Applied Mathematics and Mechanics 62, 927-937. 
[25] Pukhnachov, V.V. 1999 On a problem of viscous strip deformation with a free boundary. C. R. Acad. Sci. Paris 328, Série 1, 357-362.

[26] Shulman, Z.P. \& Kordonski V.I. 1982 Magneto-rheological Effect. Nauka i Technika. Minsk (in Russian).

[27] Shulman, Z.P. \& Nosov B.M. 1985 Rotation of Nonconducting Bodies in Electrorheological Suspensions. Nauka i Technika. Minsk (in Russian).

[28] Schwartz, L. 1967 Analyse Mathématique 1. Hermann.

[29] Solonnikov, V.A. 1977 The solvability of a problem on motion of viscous incompressible fluid bounded by a free surface. Izv. Akad. Nauk. SSSR, Ser. Mat. 41, 1388-1424 (in Russian).

[30] Solonnikov, V.A. 1984 The solvability of the problem on the evolution of an isolated volume of a viscous incompressible capillary liquid. Zap. Nauchn. Seminarov Leningr, Optiko-Mekh. Inst. 140, 179-186 (in Russian).

[31] Vainberg, M.M. 1964 Variational Methods for the Study of Nonlinear Operators. Holden Day, San Francisco.

[32] Whittle, M., Atkin, R.J. \& Bullough, W.A. 1995 Fluid dynamic limitations on the performance of an electrorheological clutch. Journal of Non-Newtonian Fluid Mechanics 57, 61-81.

[33] Whittle, M., Atkin, R.J. \& Bullough, W.A. 1999 Dynamics of a radial electrorheological clutch. International Journal of Modern Physics B 13, 2119-2126.

[34] Whittle, M., Atkin, R.J. \& Bullough, W.A. 1996 Dynamics of an electrorheological valve. International Journal of Modern Physics B 10, 2933-2950.

[35] Whittle, M., Firoozian, R., Peel, D.J. \& Bullough, W.A. 1995 Electrorheological relaxation times derived from pressure response experiments in the flow mode. Journal of Non-Newtonian Fluid Mechanics $\mathbf{5 7}, 1-25$. 